\title{
Topography-guided photo-refractive keratectomy followed by corneal collagen cross-linking for keratoconus
}

\author{
Magdy Ezzat Khallaf, Mohamed Ahmed El-Mallah, Rasha Hassan Ismail \\ Department of Ophthalmology, Faculty of Medicine, Al-Azhar University \\ *Corresponding Author: Rasha Hassan Ismail, Phone No.: (+2)01200665575, E-mail: rashaesmail@ yahoo.com
}

\begin{abstract}
Background: $\mathrm{KC}$ is a bilateral, progressive, non-inflammatory corneal degeneration. Corneal deformation and thinning causes irregular astigmatism and leads to visual impairment. Aim of the work: this study aimed to evaluate simultaneous topography-guided partial PRK and CXL as a therapeutic intervention in patients with KC. Methodology: the present study was designed to evaluate simultaneous topography-guided partial PRK and CXL as a therapeutic intervention in patients with KC. It included 25 keratoconic eyes of 17 patients; 8 cases were bilateral and 9 cases were unilateral. All included cases underwent full history taking and ophthalmologic examinations for preoperative evaluation and postoperative assessment. Follow up was carried out at first postoperative day and at 1, 3 and 6 months post-operatively. Results: when comparing K1, K2, thinnest location and BCVA at 1,3 and 6 months postoperatively to the corresponding values at first postoperative day, there was non-significant difference at any point of time. Conclusion: simultaneous topography-guided partial PRK and CXL as a therapeutic intervention in patients with keratoconus is an effective without significant complications; thus, it had a good efficacy, stability over time and safety.
\end{abstract}

Keywords: KC, ICRS, CXL

\section{Introduction}

Keratoconus (KC) is a progressive corneal disorder that can lead to severe vision deterioration through the development of irregular astigmatism and corneal scarring. Thus far, techniques that have been employed to improve vision in patients with $\mathrm{KC}$ include the use of spectacles, rigid gas permeable contact lens, intracorneal ring segment (ICRS) implantation, and in the most severe cases, lamellar or penetrating keratoplasty ${ }^{(\mathbf{1})}$. It has been shown that the progression of the disorder can be impeded by riboflavin-ultraviolet A (UVA) corneal collagen cross-linking $(\mathrm{CXL})^{(2)}$. The application of CXL is capable of stabilizing the cornea in $\mathrm{KC}$ and in postoperative refractive ectasia for up to 5 years after the procedure, but topography and visual outcomes of treated patients show only minimal improvement ${ }^{(3)}$. The irregular astigmatism in $\mathrm{KC}$ can be confronted with the use of customized laser ablations that restore the refractive properties of the anterior corneal surface. The application of

\section{Patients and methods Patients:}

The present study included 25 keratoconic eyes. They were from 17 patients; 8 cases were bilateral and 9 cases were unilateral. This study was conducted at Ophthalmology Department; AlAzhar Faculty of Medicine and International Ophthalmology Hospital (New Damietta) during customized topography-guided surface ablation treatment (T.CAT) has been reported in patients with stable or subclinical $\mathrm{KC}$ with promising visual outcomes ${ }^{(4)}$. The disadvantage of ablative procedures is that tissue removal might lead to further destabilization of corneal biomechanics and progression of the ectatic disorder. Until recently, simultaneous topography-guided Photo-refractive Keratectomy (PRK) followed by CXL seems to be the most effective approach for optimum results in the treatment of $\mathrm{KC}$ patients, as it is capable of offering functional vision with stabilization of the ectatic disorders ${ }^{(\mathbf{1})}$.

\section{Aim of the work}

The aim of this work was to evaluate simultaneous topography-guided partial PRK and CXL as a therapeutic intervention in patients with $\mathrm{KC}$.

the period from January 2014 till June 2018. The study was approved by the Ethics Board of AlAzhar University.

Before inclusion in the study, all patients were offered explanation and all information needed about the procedure, indication, technique, post-operative expectations and possibility of complications. Then, an informed consent was obtained from the patient. $\mathrm{KC}$ was diagnosed using 
computerized videokeratography (Keratograph, Oculus Optikgerate $\mathrm{GmbH}$ ) and ultrasound (US) pachymetry (Corneo Gage Plus, Sonogage, Inc.).

\section{Pre- and post-operative evaluation:}

History

The patients were asked about:

- First notice of change of visual acuity.

- The history of any previous eye disease, progression of the condition in the form of diminution of vision, time of spectacle change, contact lens wear and tolerability.

- Visual requirements for work and lifestyle, satisfaction with current visual acuity and postoperative visual expectations.

Ophthalmological examination

With emphasis on:

Visual acuity:

- Uncorrected and BCVA was determined on Snellen's chart.

\section{Refraction:}

- Cycloplegic refraction objectively by autorefractometer 800 Auto KeratoRefractometer; Topcon) and streak retinoscopy (KEE 1302-P-1010; Keeler).

- Manifest refraction was determined.

Slit lamp biomicroscopy: (Topcon)

- For signs of KC like brominent corneal nerves, stromal thinning, conical protrusion, Fleischer's ring, Vogt's striae, and corneal scarring.

Fundus examination using:

- Indirect with slit lamp and 90 lens or 78 lens.

- Keeler indirect ophthalmoscope to exclude retinal pathology.

- Keeler direct ophthalmoscope to detect Charleaux's sign or oil droplet sign.

Intraocular pressure measurement using:

- Shinnippon applanation tonometer to detect any rise of IOP.

\section{Corneal Imaging}

- Using the Oculus Pentacam with Scheimpflug imaging (OCULUS Inc., USA \#112， $2125196^{\text {th }}$ Street SW, Lynnwood, WA 98036, Toll free 1888-284-8004, Fax +1-425-670-0742.

\section{Surgical procedure}

The surgical procedure was done according to Athens protocol described by Kanellopoulos ${ }^{(5)}$.

- Phototherapeutic keratectomy at a $6.5 \mathrm{~mm}$ Results corneal epithelium. Then the partial topography-guided PRK laser treatment was applied.

- Mitomycin-C $0.02 \%$ was applied over the ablated tissue for 20 seconds, followed by irrigation with $20 \mathrm{ml}$ of chilled balanced salt solution.

- For the next 10 minutes, $0.1 \%$ riboflavin sodium phosphate ophthalmic solution was applied topically every 2 minutes.

- The corneal stroma rapidly soaks up the solution, because the central Bowman's membrane has been removed.

- Following the initial administration of riboflavin, UV-A light is used to project 3 $\mathrm{mW} / \mathrm{cm}^{2}$ of radiance at a distance of $2.5 \mathrm{~cm}$ onto the surface of the cornea for 30 minutes.

- A bandage contact lens was placed on the cornea at the completion of the combined procedure. After CXL, the patient was instructed to use topical antibiotic four times per day for the first 10 days and steroids (prednisolone acetate 1\%) four times per day for 30 days.

- Protection from all-natural light with sunglasses was encouraged along with $1000 \mathrm{mg}$ of oral vitamin $\mathrm{C}$ daily for 60 days. The bandage contact lens was removed after complete re-epithelialization, which usually occurs around (3-5days).

Postoperative follow up visits were done at first postoperative day, 1; 3 and 6 months. On the first postoperative day, slit lamp examination was done; Snelln's UCVA and BCVA measurements were done in all times of postoperative measurements. Corneal Pentacam was done at 3 and 6 months postoperatively.

\section{Statistical analysis of data:}

The collected data were organized, tabulated and statistically analyzed using statistical package for social science. For quantitative data, arithmetic mean, standard deviation (SD), minimum and maximum values were calculated; while categorical data were represented as relative frequency of observations and percentage distribution. Paired samples student $t$ test was used to compare preoperative values with postoperative values and compared at different postoperative points of times to the first postoperative measurements. P-value equal to or less than 0.05 was statistically significant and marked by asterisk (*).

\section{After follow up of all patients for 6 months, data was collected and analyzed as follow:}


Table 1: gender distribution in studied cases

\begin{tabular}{||c|c|c|c||}
\hline \hline \multicolumn{2}{|c|}{} & n. & $\%$ \\
\hline \multirow{3}{*}{ Gender } & Male & 6 & 35.3 \\
\cline { 2 - 4 } & Female & 11 & 64.7 \\
\cline { 2 - 4 } & Total & 17 & 100.0 \\
\hline
\end{tabular}

As regard to gender distribution; the present study included 17 cases; 6 of them (35.3\%) were males and 11 of them $(64.7 \%)$ were females.

Table 2: age distribution in studied cases

\begin{tabular}{||l|c|}
\hline \hline & Age (years) \\
\hline Minimum & 18.00 \\
\hline Maximum & 25.00 \\
\hline Mean \pm SD & $21.41 \pm 2.26$ \\
\hline
\end{tabular}

As regard to age, it was ranged from 18 to 25 years with a mean of 21.41 years and standard deviation of 2.26 years.

Table 3: laterality and side distribution of $\mathrm{KC}$ in studied cases

\begin{tabular}{||c|l|c|c||}
\hline \hline \multirow{2}{*}{ Laterality } & & No. & $\%$ \\
\hline \multirow{2}{*}{ Side } & Unilateral & 9 & 52.9 \\
\cline { 2 - 4 } & Bilateral & 8 & 47.1 \\
\cline { 2 - 4 } & Right & 10 & 40.0 \\
\cline { 2 - 4 } & Left & 15 & 60.0 \\
\hline
\end{tabular}

As regard to laterality in the studied cases; 9 patients (52.9\%) had unilateral $\mathrm{KC}$ and 8 patients (47.1\%) had bilateral KC. The KC was on the right in 10 eyes (40.0\%) and on the left in 15 eyes (60.0\%). Table 4: comparison between pre-and first day post-operative values of $\mathrm{K} 1$ in the studied cases

\begin{tabular}{||c|l|c|c|c|c||}
\hline \hline \multirow{2}{*}{ K1 } & & Mean \pm & S. D & Minimum & Maximum \\
\hline \multirow{2}{*}{ Statistics } & Preoperative & $42.68 \pm$ & 2.68 & 38.40 & 47.23 \\
\cline { 2 - 6 } & Postoperative & $41.59 \pm$ & 2.30 & 37.50 & 45.40 \\
\hline & Paired (t) & \multicolumn{4}{|c|}{-7.24} \\
\cline { 2 - 6 } & P value & \multicolumn{4}{|c|}{$0.001^{*}$} \\
\hline
\end{tabular}

As regard K1; preoperatively, it ranged from 38.40 to 47.23 with a mean of $42.68 \pm 2.68$; postoperatively at the first day, it ranged from 37.50 to 45.40 with a mean of $41.59 \pm 2.30$; and there was significant decrease of $\mathrm{K} 1$ at postoperative day when compared to their preoperative values.

Table 5: comparison between pre-and first day post-operative values of $K 2$ in the studied cases

\begin{tabular}{||c|l|c|c|c|c||}
\hline \hline \multirow{2}{*}{ K2 } & & Mean \pm & S. D & Minimum & Maximum \\
\hline \multirow{2}{*}{ Statistics } & Preoperative & $44.73 \pm$ & 2.28 & 40.10 & 49.70 \\
\cline { 2 - 6 } & Postoperative & $42.55 \pm$ & 2.56 & 38.20 & 49.0 \\
\cline { 2 - 6 } & Paired (t) & \multicolumn{4}{|c|}{-66.92} \\
\cline { 2 - 5 }
\end{tabular}

Regarding K2 preoperatively, it ranged from 40.10 to 49.70 with a mean of $44.73 \pm 2.28$; at the first day postoperatively, K2 ranged from 38.20 to 49.0 with a mean of $42.55 \pm 2.56$; and there was significant decrease of $\mathrm{K} 2$ at first postoperative day when compared to their preoperative values.

Table 6: comparison between pre-and first day post-operative values of thinnest location in the studied cases

\begin{tabular}{||c|l|c|c|c|c||}
\hline \hline & & Mean \pm & S. D & Minimum & Maximum \\
\hline \multirow{2}{*}{ Thinnest location } & Preoperative & $477.16 \pm$ & 28.44 & 426.00 & 552.00 \\
\cline { 2 - 6 } & Postoperative & $408.56 \pm$ & 36.41 & 338.00 & 487.00 \\
\hline \multirow{2}{*}{ Statistics } & Paired (t) & \multicolumn{4}{|c|}{-13.72} \\
\cline { 2 - 6 } & P value & \multicolumn{4}{|c|}{$<0.001^{*}$} \\
\hline
\end{tabular}

As regard to thinnest location preoperatively, it ranged from 426 to 552 with a mean of 477.16 \pm 28.44 ; while at first postoperative day, it ranged from 338 to 487 with a mean of $408.56 \pm 36.41$ and there was a significant decrease of thinnest location at first postoperative day when compared to preoperative values.

Table 7: comparison between pre-and first day post-operative values of BCVA in the studied cases

\begin{tabular}{|l|l|l|l|l|c||}
\hline \hline & Mean \pm & S. D & Minimum & Maximum \\
\hline
\end{tabular}




\begin{tabular}{||c|l|c|c|c|c||}
\hline \multirow{2}{*}{ BCVA } & Preoperative & $0.20 \pm$ & 0.17 & 0.05 & 0.60 \\
\cline { 2 - 6 } & Postoperative & $0.93 \pm$ & 0.18 & 0.60 & 1.20 \\
\hline \multirow{2}{*}{ Statistics } & Paired (t) & \multicolumn{4}{|c|}{16.89} \\
\cline { 2 - 5 } & P value & \multicolumn{4}{|c|}{$<0.001^{*}$} \\
\hline
\end{tabular}

As regard to BCVA preoperatively, it was ranged from 0.05 to 0.60 with a mean of $0.20 \pm 0.17$; while at the first day postoperatively, it ranged from 0.60 to 1.20 with a mean of $0.93 \pm 0.18$ and there was a significant increase of VA at the first postoperative day when compared to their preoperative values.

Table 8: K1 at 1, 3, 6 months compared to their values at the first postoperative day

\begin{tabular}{||l|l|c|c|c|c||}
\hline \hline & & Mean \pm & S. D & Minimum & Maximum \\
\hline \multirow{3}{*}{ K1 } & First postoperative day & $41.59 \pm$ & 2.30 & 37.50 & 45.40 \\
\cline { 2 - 6 } & At 1 months PO & $41.42 \pm$ & 2.93 & 37.50 & 48.80 \\
\cline { 2 - 6 } & At 3 months PO & $41.42 \pm$ & 2.92 & 37.50 & 48.70 \\
\cline { 2 - 6 } & At 6 months PO & $41.41 \pm$ & 2.92 & 37.50 & 48.60 \\
\hline \multirow{2}{*}{ Statistics } & Paired t & \multicolumn{3}{|c|}{$\mathrm{t} 1=1.42, \quad \mathrm{t} 2=1.39, \mathrm{t} 3=1.43$} \\
\cline { 2 - 6 } & P values & \multicolumn{2}{|c|}{$\mathrm{P} 1=0.17(\mathrm{NS}), \mathrm{p} 2=0.18(\mathrm{NS}), \mathrm{p} 3=0.16(\mathrm{NS})$} \\
\hline
\end{tabular}

When comparing $\mathrm{K} 1$ at 1,3 and 6 months postoperatively to the corresponding values at first postoperative day, there was non-significant difference at any point of time.

Table 9: K2 at 1, 3, 6 months compared to their values at the first postoperative day

\begin{tabular}{||l|l|c|c|c|c||}
\hline \hline & & Mean \pm & S. D & Minimum & Maximum \\
\hline \multirow{3}{*}{ K2 } & First postoperative day & $42.55 \pm$ & 2.56 & 38.20 & 49.0 \\
\cline { 2 - 6 } & At 1 months PO & $43.28 \pm$ & 3.61 & 38.10 & 54.90 \\
\cline { 2 - 6 } & At 3 months PO & $43.26 \pm$ & 3.62 & 38.20 & 54.90 \\
\cline { 2 - 6 } & At 6 months PO & $43.27 \pm$ & 3.62 & 38.20 & 54.90 \\
\hline \multirow{2}{*}{ Statistics } & Paired t & \multicolumn{3}{|c|}{$\mathrm{t} 1=1.39}$, & $\mathrm{t} 2=1.14, \mathrm{t} 3=1.43$ \\
\cline { 2 - 6 } & P values & P1=0.17(NS), p 2 =0.26(NS), p 3 = 0.16(NS) \\
\hline
\end{tabular}

When comparing $\mathrm{K} 2$ at 1,3 and 6 months postoperatively to the corresponding values at first postoperative day, there was a non-significant difference at any point of time.

Table 10: thinnest location at $1,3,6$ months compared to their values at the first postoperative day

\begin{tabular}{||c|l|c|c|c|c||}
\hline & & Mean \pm & S. D & Minimum & Maximum \\
\hline \multirow{4}{*}{ Thinnest location } & First postoperative day & $408.56 \pm$ & 36.41 & 338.00 & 487.00 \\
\cline { 2 - 6 } & At 1 months PO & $408.28 \pm$ & 36.48 & 338.00 & 487.00 \\
\cline { 2 - 6 } & At 3 months PO & $408.20 \pm$ & 36.46 & 338.00 & 487.00 \\
\cline { 2 - 6 } & At 6 months PO & $408.28 \pm$ & 36.45 & 338.00 & 487.00 \\
\hline \multirow{2}{*}{ Statistics } & Paired t & \multicolumn{3}{|c|}{$\mathrm{t} 1=1.15, \mathrm{t} 2=1.36, \mathrm{t} 3=1.42$} \\
\cline { 2 - 5 } & P values & \multicolumn{2}{|c|}{ P1=0.25(NS), p 2=0.18(NS), p 3 = 0.17(NS) } \\
\hline
\end{tabular}

When comparing thinnest location at 1, 3 and 6 months postoperatively to the corresponding values at first postoperative day, there was non-significant difference at any point of time.

Table 11: BCVA at 1, 3, 6 months compared to their values at the first postoperative day

\begin{tabular}{||l|l|c|c|c|c||}
\hline \hline & & Mean \pm & S. D & Minimum & Maximum \\
\hline \multirow{3}{*}{ BCVA } & First postoperative day & $0.92 \pm$ & 0.18 & 0.60 & 1.20 \\
\cline { 2 - 6 } & At 1 months PO & $0.94 \pm$ & 0.17 & 0.60 & 1.20 \\
\cline { 2 - 6 } & At 3 months PO & $0.94 \pm$ & 0.17 & 0.60 & 1.20 \\
\cline { 2 - 6 } & At 6 months PO & $0.94 \pm$ & 0.17 & 0.60 & 1.20 \\
\hline \multirow{2}{*}{ Statistics } & Paired t & \multicolumn{4}{|c|}{$\mathrm{t} 1=1.44, \mathrm{t} 2=1.44, \mathrm{t} 3=1.44$} \\
\cline { 2 - 6 } & P values & \multicolumn{2}{|c|}{$\mathrm{P} 1=0.16(\mathrm{NS}), \mathrm{p} 2=0.16(\mathrm{NS}), \mathrm{p} \mathrm{3}=0.16(\mathrm{NS})$} \\
\hline
\end{tabular}

When comparing BCVA at 1, 3 and 6 months postoperatively to the corresponding values at first postoperative day, there was non-significant difference at any point of time.

\section{Discussion:}

$\mathrm{KC}$ is the most common corneal ectatic disease. It is a chronic progressive eye condition in which the cornea deforms to a more conical shape causing visual impairment. For the age group 1044 years, the prevalence of $\mathrm{KC}$ is 57 per 100000 in
Caucasians, but over four-fold higher in people originating from the Indian subcontinent. Conventionally spectacles, contact lenses, and corneal transplantation are the mainstay of treatment ${ }^{(6)}$.In brief, this CXL procedure leads to 
Topography-guided photo-refractive keratectomy followed by corneal collagen...

photo-oxidation leading to additional covalent bonds between and within collagen fibrils of the cornea, which increases corneal stiffness, stabilizes the $\mathrm{KC}$ and, in some cases, improves refractive and topographic features ${ }^{(7)}$. The CXL technique has evolved rapidly over the last decade. A study reported the use of pharmacological agents to loosen the epithelium before instillation of riboflavin ${ }^{(8)}$, iontophoretic experiments to enhance the riboflavin permeability, partial disruption of epithelium ${ }^{(9)}$ and even CXL with intact epithelium ${ }^{(8)}$.The present study was designed to evaluate simultaneous topography-guided partial PRK and CXL as a therapeutic intervention in patients with KC. It included 25 keratoconic eyes of 17 patients; 8 cases were bilateral and 9 cases were unilateral. The present study included 17 cases; 6 of them $(35.3 \%)$ were males and 11 of them $(64.7 \%)$ were females. Age ranged from 18 to 25 years with a mean of 21.41 years and standard deviation of 2.26 years. These results are comparable to those reported by De Bernardo et al. ${ }^{(10)}$ who reported that, thirty-six eyes of 36 patients ( 28 men) with a mean age of $24.5 \pm 5$ years (range from 12 to 33 ) with a progressive $\mathrm{KC}$, documented by refraction and corneal topography in the last 6 months were included in the study. In addition, Goldich et al. ${ }^{(11)}$ reported that, seventeen eyes of 17 patients (12males, 5 females) aged $27.3 \pm 5.1$ years were included. Results of our study indicated that CXL can achieve disease stabilization in eyes of patients with KC. The CXL treatment significantly reduced the maximum and minimum corneal curvatures and the curvature at the apex of the $\mathrm{KC}$ in the eyes in our study population. The CDVA was also significantly improved, which can be attributed to the reductions in corneal astigmatism and corneal distortion and to a better fit of the hard contact lenses enabled by the regularization of the corneal surface. These findings confirm the reported outcomes in a larger case series, which observed 241 eyes of KC patients.

In our study there was a statistically significant improvement was also observed in corneal astigmatism, CDVA and maximum $\mathrm{K}$. They reported that, at the end of follow up after CXL, 149 (91.4\%) eyes of 114 patients had a clear cornea without stromal haze (control group), whereas 14 eyes $(8.6 \%)$ of 13 patients developed clinically significant stromal haze (haze group). Preoperatively, the mean keratometry $(\mathrm{K})$ value of the apex was $62.1+/-13.8$ diopters (D) in the control group and $71.1+/-13.2 \mathrm{D}$ in the haze group $(\mathrm{P}=.02)$. The mean corneal thickness before the procedure was $478.1+/-52.4$ mum in the control group and 420.0+/-33.9 mum in the haze group $(\mathrm{P}=.001)$. Uncorrected visual acuity and BSCVA, which were similar between groups preoperatively $(\mathrm{P}=.59$ and $\mathrm{P}=.75$, respectively), were improved in the control group $(\mathrm{P}=.023$ and $\mathrm{P}=.001$, respectively), but reduced in the haze group $(\mathrm{P}=.012$ and $\mathrm{P}=.004$, respectively) postoperatively. Caporossi et $\boldsymbol{a l} \cdot{ }^{\left({ }^{(13)}\right.}$ found no clinically or statistically significant improvements in UCVA and BCVA24 months after the procedure, a worsening of the maximum $\mathrm{K}$ value, a decrease in thinnest point, no significant variation in coma aberrations. The median baseline UDVA was 0.4 Snellen lines. An improvement in UDVA, although not statistically significant, was recorded at 6 months (median 0.45 Snellen line; $\mathrm{P}=0.13$ ) and lasted until the $12^{\text {th }}$ month. At the 18 -month followup, the UDVA was worse, returning to baseline values (median 0.4 Snellen line).

In our study the median baseline maximum $\mathrm{K}$ value was $48.59 \mathrm{D}$ (range 46.83 to $59.11 \mathrm{D}$ ). The maximum topographic curvature (maximum $\mathrm{K}$ ) showed no statistically significant increase 12 months after treatment (median $49.19 \mathrm{D}$; range 44.5 to $61.42 \mathrm{D}$ ). This trend increased at 18 months with a statistically significant worsening at 24 months (median 50.14 D). As regard to thinnest location preoperatively, it ranged from 426 to 552 with a mean of $477.16 \pm 28.44$; while at first postoperative day, it ranged from 338 to 487 with a mean of $408.56 \pm 36.41$; and there was significant decrease of thinnest location at first postoperative day when compared to preoperative values. These results are comparable to those reported by Raiskup et al. ${ }^{(12)}$ who reported that the mean corneal thickness at surgery was $488 \pm 46 \mathrm{~mm}$, which fulfilled the treatment criteria. They added that the mean apical keratometry $(\mathrm{K})$ value was 61.5 diopters (D) preoperatively and $55.3 \mathrm{D} 10$ years postoperatively; the decrease was statistically significant $(\mathrm{P}<.001)$. In our study, the mean values for maximum K (53.2 D and 49.56 D, respectively) and minimum $\mathrm{K}$ (47.5 D and 45.5 D, respectively) were also significantly lower $(\mathrm{P}<.001)$. The preoperative and postoperative CDVA were statistically significantly different $(\mathrm{P}=.002)$. The mean CDVA improved by $0.14 \log$ MAR over preoperatively; the change was statistically significant $(\mathrm{P}=.002)$.The nature of the initial continuous flattening still remains unclear, but plausibly at the longer term, inherent pathological stromal remodeling leads to reversal of achieved cross-linking stabilization effects and renewal of $\mathrm{KC}$ progression. In the present study, no complications were reported during follow up 
period. These results agree with those reported by Raiskup et al. ${ }^{(12)}$ who reported that relevant complications, such as prolonged reepithelialization, infection, limbal stem cell insufficiency, and endothelial decompensation, were not observed during the 10-year follow-up. Koller et al. ${ }^{(14)}$ examined 117 participants 12 months after CXL was performed, found permanent corneal scarring in $2.8 \%$ of cases. In Raiskup et al. ${ }^{(12)}$ study of the CXL treatment and 12-month follow-up of 163 eyes, a slightly higher proportion of eyes had persistent vision-impairing deep stromal scarring (8.6\%). That study also reported an overall increased risk for scarring in eyes having very high preoperative apical $\mathrm{K}$ values for the $\mathrm{KC}$ and in eyes with thinner corneas, as typically seen in an advanced stage of $\mathrm{KC}$.

In conclusion results of the present study confirm that CXL is effective in patients with $\mathrm{KC}$, without significant complications; thus, it had a good efficacy, stability over time and safety. Simultaneous topography-guided partial PRK and CXL as a therapeutic intervention in patients with keratoconus is an effective without significant complications; thus, it had a good efficacy, stability over time and safety. Results of this study revealed that both $\mathrm{K} 1$ and $\mathrm{K} 2$ were significantly reduced at the first day postoperatively and become stable overtime till the end of the six months of follow up. In addition, thinnest location and BCVA at 1, 3 and 6 months postoperatively to the corresponding values at first postoperative day, there was nonsignificant difference at any point of time.

\section{References}

1. Kymionis GD, Portaliou DM, Panagopoulou SI et al. (2012): Corneal collagen cross-linking with riboflavin and ultraviolet-A irradiation in patients with thin corneas. Am. J. Ophthalmol., 153: 24 28.

2. Wollensak G, Spoerl $\mathbf{E}$ and Seiler $\mathbf{T}$ (2003): Riboflavin/ultravioleta- induced collagen crosslinking for the treatment of KC. Am. J. Ophthalmol., 135(5): 620-627.

3. Raiskup F, Theuring A, Pillunat LE et al. (2015): Corneal collagen crosslinking with riboflavin and ultraviolet-A light in progressive KC: Ten-year results. J. Cataract. Refract. Surg., 41:41-46.

4. Koller T, Schumacher S, Fankhauser F et al. (2013): Riboflavin/ultraviolet A crosslinking of the paracentral cornea. Cornea, 32: 165-168.

5. Kanellopoulos AJ. (2009): Comparison of sequential vs same-day simultaneous collagen cross-linking and topographyguided PRK for treatment of KC. J. Refract. Surg., 25: 812-818.

6. Shalchi $Z$, Wang $X$ and Nanavaty MA (2015): Safety and efficacy of epithelium removal and transepithelial corneal collagen crosslinking for KC. Eye, 29: 1529.

7. Kranitz K, Kovacs I, Mihaltz $\mathrm{K}$ et al. (2012): Corneal changes in progressive KC after cross-linking assessed by Scheimpflug camera. J. Refract. Surg., 28(9): 645-649.

8. Filippello M, Stagni E and O'Brart D (2012): Transepithelial corneal collagen crosslinking: bilateral study. J. Cataract. Refract. Surg., 38(2): 283-291.

9. Samaras KE and Lake DB (2010): Corneal collagen cross linking (CXL): a review. Int. Ophthalmol. Clin., 50(3): 89100.

10. De Bernardo $M$ and Rosa $N$ (2018): Repeatability and agreement of orbscan II, pentacam HR, and galilei tomography systems in corneas with keratoconus. Am. J. Ophthalmol., 186:166-176.

11. Goldich Y, Barkana Y, Lior OW et al. (2014): Corneal collagen cross-linking for the treatment of progressive $\mathrm{KC}$ : 3-year prospective outcome. Can. J. Ophthalmol., 49:54-59.

12. Raiskup-Wolf F, Hoyer A, Spoerl E et al. (2008): Collagen crosslinking with riboflavin and ultraviolet-A light in KC: longterm results. J. Cataract Refract. Surg., 34:796-801.

13. Caporossi A, Mazzotta C, Paradiso AL et al. (2013): Transepithelial corneal collagen crosslinking for progressive $\mathrm{KC}$ : 24-month clinical results. J. Cataract Refract. Surg., 39: 1157-1163.

14. Koller $T$, Mrochen $M$ and Seiler $T$ (2009): Complication and failure rates after corneal crosslinking. J. Cataract Refract. Surg., 35:1358-1362. 